\title{
Watching the infant brain learn words: effects of vocabulary size and experience
}

\author{
Debra L. Mills ${ }^{\mathrm{a}}$, Kim Plunkett ${ }^{\mathrm{b}, *}$, Chantel Prat ${ }^{\mathrm{c}}$, Graham Schafer ${ }^{\mathrm{d}}$ \\ a Emory University, Atlanta, GA 30322, UK \\ b Oxford University, South Parks Road, Oxford OX1 3UD, UK \\ ${ }^{\mathrm{c}}$ University of California, Davis, USA \\ ${ }^{\mathrm{d}}$ University of Reading, Whiteknights, P.O. Box 217, UK
}

Received 1 September 2003; received in revised form 1 May 2004; accepted 1 July 2004

\begin{abstract}
Previous investigations comparing auditory event-related potentials (ERPs) to words whose meanings infants did or did not comprehend, found bilateral differences in brain activity to known versus unknown words in 13-month-old infants, in contrast with unilateral, left hemisphere, differences in activity in 20-month-old infants. We explore two alternative explanations for these findings. Changes in hemispheric specialization may result from a qualitative shift in the way infants process known words between 13 and 20 months. Alternatively, hemispheric specialization may arise from increased familiarity with the individual words tested. We contrasted these two explanations by measuring ERPs from 20-month-old infants with high and low production scores, for novel words they had just learned. A bilateral distribution of ERP differences was observed in both groups of infants, though the difference was larger in the left hemisphere for the high producers. These findings suggest that word familiarity is an important factor in determining the distribution of brain regions involved in word learning. An emerging left hemispheric specialization may reflect increased efficiency in the manner in which infants process familiar and novel words.
\end{abstract}

(c) 2004 Elsevier Inc. All rights reserved.

Keywords: Language acquisition; Vocabulary spurt; Word learning; Event related potentials; Lexical development; Semantic development; Familiarity

\footnotetext{
* Corresponding author. Tel.: +44 1854 27198; fax: +44 1865310447.

E-mail address: kim.plunkett@pay.ox.ac.uk (K. Plunkett).
} 


\section{Introduction}

The second year of life heralds dramatic advances in the infant's expressive and receptive language abilities. Yet little is known about the development of the underlying brain systems that permit this development to occur. In a recent study, Mills, Coffey-Corina, and Neville (1997) examined auditory event-related potentials (ERPs) to words whose meanings the infants comprehended and compared them with ERPs to words the infants did not comprehend. At 13-17 months, ERP amplitude differences between comprehended and unknown words were bilateral, i.e., observed over both the left and right hemispheres, and broadly distributed over anterior and posterior regions. In contrast, at 20 months, these effects were limited to temporal and parietal regions of the left hemisphere (see also, Mills, Prat, Zangl, Stager, Neville, \& Werker, in press for a replication of this age-related effect). The authors suggested that differences in ERPs between the two age groups might be linked to the remarkable changes in early lexical development that typically occur between 13 and 20 months. Specifically, they proposed that hemispheric specialization of activity differences between comprehended and unknown words might result from a qualitative shift in the way infants process known words.

Various theoretical accounts have been offered to explain the rapid linguistic development observed during the second year, such as the occurrence of a naming insight (McShane, 1979), changes in categorization abilities (Gopnik \& Meltzoff, 1987), object permanence skills (Corrigan, 1978), and the emergence of speech segmentation abilities (Plunkett, 1993). Other approaches have argued that qualitative shifts in processing are not needed to explain linguistic development in the second year. For example, Plunkett, Sinha, Møller, and Strandsby (1992) argued that non-linearities in vocabulary growth could be explained in terms of the strengthening of word-object associations with age. This view implies that the age-related differences observed by Mills et al. (1997) may have resulted from the different amounts of experience with the words used in the study across the two age groups. That is, 20-month-olds will have had more opportunities to hear and use the 'known' words than 13-month-olds. According to Plunkett et al. (1992), increased experience with a word leads directly to a change in the way that word is processed and thus to age-related changes in patterns of brain activity. Such changes need not, however, reflect changes in underlying processing mechanism.

ERP signatures associated with known and unknown words have also been studied in late talkers and a group of age-matched controls (Mills, Conboy, \& Paton, in press). Twentymonth and 28- to 30-month-old late talkers, whose vocabulary sizes matched the 13- to 20-month-old typically developing infants in the earlier studies, showed a change in the lateral distribution of brain activity to known and unknown words, i.e., from bilateral to left lateralized, as a function of increasing vocabulary size. These results identify language experience, rather than age, as a determining factor for increasing cerebral specialization for language.

Conboy, 2002 and Conboy and Mills, 2000 examined ERPs to known and unknown words in 20-month-olds learning both Spanish and English simultaneously. The results showed that the distribution of ERP amplitude differences between known and unknown words depended on the number of words the child had in each language separately. That is, ERP differences between known and unknown words were more focally distributed to words 
in the child's dominant language. These findings lend further support to the hypothesis that experience with learning language, rather than age or brain maturation, is the important factor for increasing cerebral specialization for language.

In the present study, we examine the hypothesis that it is experience with individual word-object associations rather than overall vocabulary size that is responsible for the dynamic shifts in ERP signatures observed in the original Mills et al. (1997) and later studies. We tested this hypothesis by training a group of typically developing 19- to 20month-olds to associate two novel words with novel objects. Prior to training, we collected ERPs for two familiar words and four novel words. After training, we collected ERPs for the same acoustic stimuli. The 20-month-olds were assigned to a high or low production group. If experience with individual words is the primary determinant of increasing hemispheric specialization, both groups will show similar patterns of brain activity for novel words, i.e., bilateral patterns of activation differences. However, if level of language development as reflected in overall vocabulary size is the primary determinant of increasing hemispheric specialization, the high vocabulary group should exhibit left lateralized patterns of activation differences.

\section{Method}

\subsection{Participants}

Twenty-two normally developing infants ( 9 girls) from 17 to 21 months of age (mean = 19.4 months) participated in the study. All children were full term (>36 weeks gestation), had no known neurological diagnosis, and were from monolingual English speaking homes. Data from an additional 19 children (13 boys and 6 girls), were excluded due to refusal to wear the electrocap $(N=6)$, excessive artifact $(N=7)$, failure to complete the experiment $(N=3)$ or computer error $(N=3)$.

\subsection{Language assessment}

Within 1 week of ERP testing, parents were asked to complete the MacArthur Communicative Development Inventory (CDI), to provide an estimate of the child's vocabulary size and percentile ranking relative to other children of the same age (Fenson et al., 1993). Percentile rankings for productive vocabulary from the MacArthur CDI norms for both genders combined were used to divide the children into two groups High and Low Producers (Table 1). The CDI and a picture-pointing task were also used to check that children knew the familiar words used in the study.

\subsection{Materials}

Auditory stimuli were recorded using infant-directed speech, digitized at $16 \mathrm{kHz}$, and stored on an IBM PC computer. ERPs were collected for two familiar words (dog and cat) and four novel words (bard, wug, gaf and sarl). Novel words were assigned to two conditions: either paired or not paired with a novel object. Assignment of the words was counterbalanced across conditions and participants. 
Table 1

Descriptive information for High and Low Producers

\begin{tabular}{lccc}
\hline & Low Producers $(N=12)$ & High Producers $(N=10)$ & Total $(N=22)$ \\
\hline Age in months & $19.5(18-21)$ & $19.2(17-21)$ & $19.4(17-21)$ \\
Gender & & & \\
$\quad$ Boys & 8 & 5 & 93 \\
$\quad$ Girls & 4 & 5 & $148(8-642)$ \\
Words produced & $63(8-106)$ & $250(144-642)$ & $44.6(0-99)$ \\
Percentile rankings & $21.9(0-49)$ & $71.9(50-99)$ & \\
\hline
\end{tabular}

\subsection{Procedure}

Words were presented using a Bose speaker with a variable stimulus onset asynchrony (SOA) of approximately $2000 \mathrm{~ms}$ (range 1800-3000 ms). The speaker was located behind a curtain on a puppet theater. A puppet moved as the words were presented to maintain the child's interest. Approximately every 15 words, a battery operated toy on the front of the puppet theater was activated to reward the child for good participation.

\subsubsection{Block 1: Before training}

The two familiar and four novel words were repeated 30 times each in random order for a total of 180 trials.

\subsubsection{Block 2: Training}

One familiar and two novel words were assigned to either an object pairing condition or a repetition condition. In the paired condition, each word was paired with an object for five repetitions. For example, a puppet was used to hold up a stuffed dog and the child heard: "Look, Look at the dog, dog, dog, dog. Look, look at the dog". Immediately following, the same procedure was used for each of the novel words to-be-paired. Novel words were paired with a novel object (a boot pull or snaffle bit). Words assigned to the not-paired condition were repeated the same number of times, but not paired with an object. For example, the child saw the same puppet used in training and heard: "Listen, hear the word cat, cat, cat, cat. Listen, hear the word cat". The same procedure was repeated for the two novel-not-paired words. The entire procedure for the paired and not-paired words was repeated a second time. Thus, each paired-word was paired with an object 10 times. Each not-paired word was repeated 10 times, but not paired with an object.

\subsubsection{Block 3: After-training}

The third block always followed the training block and was identical in procedure and number of words presented to the Before-training block.

\subsection{Electrophysiological testing}

The EEG was recorded during all three experimental blocks using tin electrodes (ElectroCap International) from sites over frontal (F7 and F8), anterior temporal (50\% of the distance from F7/8 and T3/4), temporal (33\% of the distance from T3/4 to C3/4), parietal (50\% of the distance between $\mathrm{T} 3 / 4$ and $\mathrm{P} 3 / 4)$, and occipital (O1 and $\mathrm{O} 2)$, regions of the left and right 
hemispheres, and from central locations $\mathrm{Cz}$ and Pz. Additionally, the electrocculogram was recorded from electrodes placed over and under the eye to reject trials on which blinks and vertical eye movement occurred, and from left and right frontal electrodes to reject trials on which horizontal eye movement occurred. Impedances were kept below $5 \mathrm{k} \Omega$ and were balanced (within $1 \mathrm{k} \Omega$ ) across the left and right hemispheres at any given position. All electrodes were referenced to linked mastoids. We are aware of the controversies surrounding linked mastoids (see Mills, Coffey-Corina, \& Neville, 1993, for a discussion of this issue). The EEG was amplified by SA Instruments amplifiers (input impedances of $1000 \mathrm{M} \Omega$ per channel) with a band pass of $0.1-100 \mathrm{~Hz}$ and sampled continuously every $4 \mathrm{~ms}$. Averages of the EEG were conducted using 2-s epochs.

\subsection{Artifact rejection}

Artifact rejection was conducted off-line to remove trials contaminated with blinks, horizontal and vertical eye movement, and/or amplifier blocking (see Mills, et al., 1997 for more specific information). There were at least 10 artifact-free trials $($ mean $=36$ ) per experimental condition.

\subsection{Measurement of ERPs}

ERP mean amplitudes between 200 and $500 \mathrm{~ms}$ post-stimulus onset were quantified by computer relative to a $100 \mathrm{~ms}$ pre-stimulus baseline. This time window was used because previous studies have found ERP amplitudes in this window to be modulated by word meaning.

\subsection{Data analysis}

Separate ANOVAs using BMDP 4V were conducted to: (1) compare changes in the ERPs from Block 1 to Block 3 (see Section 3.1); (2) compare ERPs to the three word-types, i.e., familiar words, novel words to-be-paired and novel words not-to-be-paired, to each other before training and (3) compare ERPs to the novel-paired and novel-not-paired words after training. Preliminary examination of the data showed that over occipital regions no ERP effects were significant for any of the experimental manipulations. Therefore, these regions were not included in the analyses. Descriptions of the ANOVAs are provided in each section to increase readability. Simple effects analyses were used for planned comparisons for the high and low producer groups as noted in the section called Group differences. Hyundt-Feldt corrections were used for the repeated measures. Effect sizes were calculated for partial eta-squared $\left(\eta^{2}\right)$ to determine the proportion of total variance attributed to each significant effect $\left(\eta^{2}=\right.$ SSeffect/(SSeffect + SSerror $\left.)\right)$.

\section{Results}

\subsection{Effects of object-word pairings versus word repetitions}

The first analysis was designed to test our hypothesis that the N200-500 amplitude is linked to word meaning. Of particular interest were amplitude differences in ERPs to the 
three types of words before and after the training phase of the experiment. This comparison allowed us to examine the effects of pairing an object with a novel word versus simply repeating the word the same number of times. Numerous studies have shown that repeating a word, or any stimulus, results in increased ERP positivities (see Rugg \& Coles, 1995 for a review). If repetition affects all word types equally, then the N200-500 amplitudes should be reduced, i.e., become more positive, for all three word-types in the After-training block relative to the Before-training block. However, if the N200-500 amplitude indexes word meaning, then the amplitude of the N200-500 should increase, i.e., show a larger negative amplitude, after training for the novel words that are paired with an object.

Changes in ERP amplitudes between the Before-training versus the After-training blocks (Block 1 versus Block 3), were compared using a repeated-measures ANOVA with factors Word experience (familiar, novel-paired, novel-not-paired), Training (before, after), Hemisphere (left, right), and Electrode site (frontal, anterior temporal, temporal, parietal).

ERP mean amplitudes between 200 and $500 \mathrm{~ms}$ (N200-500) differed before and after training for the familiar, novel-not-paired, and novel-paired words as shown in Fig. 1. Consistent with previous studies showing increased positivities to repeated stimuli, ERPs to both the familiar and novel-not-paired words showed a reduced N200-500, i.e., were more positive, after training than before training. In contrast, as predicted by the hypothesis that the N200-500 is linked with word meaning, the N200-500 mean amplitude to the

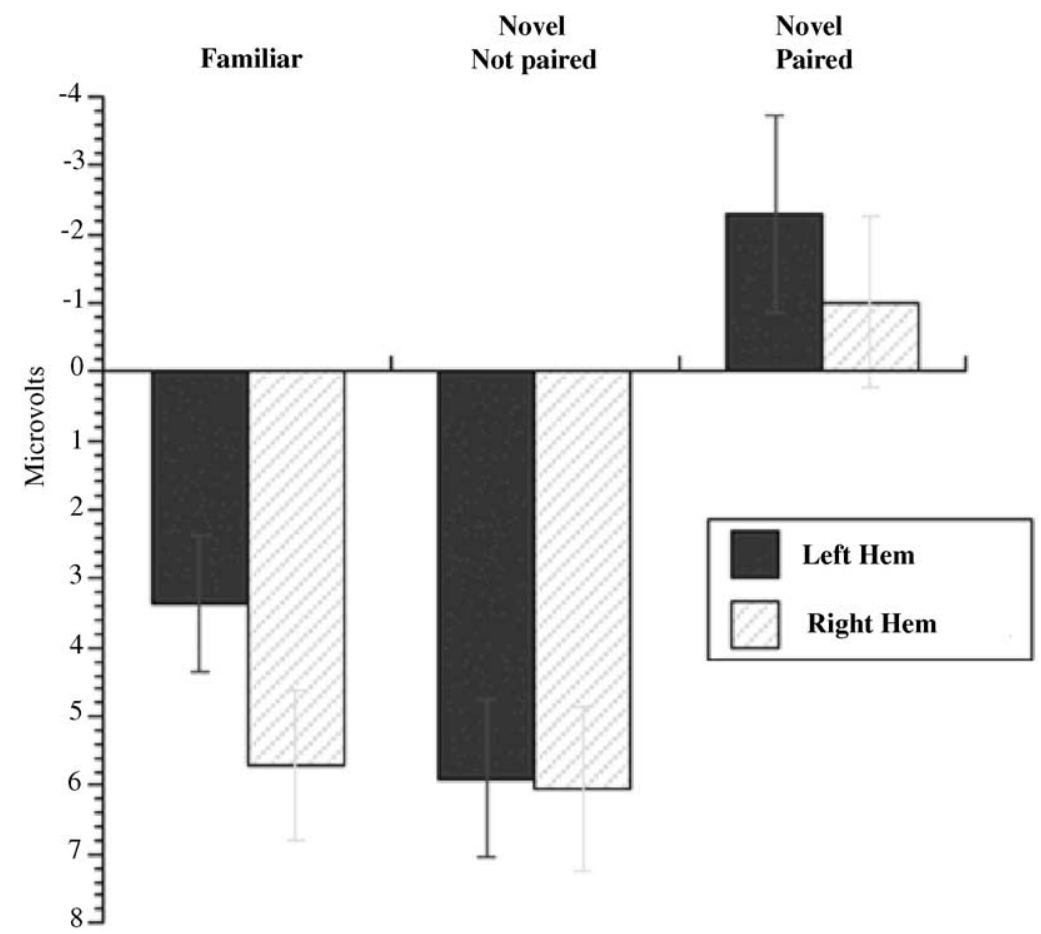

Fig. 1. Repetition effects mean area difference waves for familiar, novel-paired and novel-not-paired words. 
novel-paired words showed increased negativity after training compared to before training (Training: $F(1,21)=13.72, P=0.001, \eta^{2}=0.40$; Word experience: $F(2,42)=6.51, P$ $=0.005, \eta^{2}=0.24$; Training $\times$ Word experience: $F(2,42)=3.53, P=0.039, \eta^{2}=0.14$ ). Additionally, changes in ERP amplitudes between the Before-training versus After-training blocks tended to be larger over anterior than posterior regions (Training $\times$ Electrode site: $\left.F(3,63)=4.79, P=0.018, \eta^{2}=0.19\right)$. There were no other main effects or interactions.

\subsection{Comparisons of paired versus not paired novel words}

In a second set of analyses, we directly compared ERP amplitudes to the two types of novel words in separate ANOVAs, one for the Before-training block and one for the Aftertraining block. Because the stimuli were counterbalanced across conditions and participants, we expected no ERP amplitude differences between the two types of novel words before training. In line with our hypothesis that pairing a novel word with an object promotes word learning, we predicted that the amplitude of the N200-500 would be larger for the novel-paired than for the novel-not-paired words in the After-training block. Additionally, we examined group differences in the lateral distributions of these effects (see Section 3). ERP amplitude differences between novel-paired and novel-not-paired words for the two language groups were analyzed using the factors Language Group (high, low), Word experience (novel-paired, novel-not-paired), Hemisphere (left, right), and Electrode site (frontal, anterior temporal, temporal, parietal). Main effects and interactions with language group are presented in a separate section called Group differences.

\subsubsection{Before training}

In order to interpret differences in ERPs for the novel-paired versus the novel-not-paired after the training condition, it is important to establish that the ERPs to the two types of novel words were not different before training. Of particular interest were the effects of training on ERPs to the novel words. As predicted, prior to training, ERPs to the two types of novel words (to-be-paired and not-to-be-paired) were virtually identical (Fig. 2; ANOVA for the Before-training block, Word experience, $F(1,20)=0.11, P=0.75)$. Likewise, there were no significant differences in ERPs between the novel and familiar words (ANOVA for the Before-training block, Word experience, $F(1,20)=0.30, P=0.59)$.

\subsubsection{After training}

As predicted, pairing the novel words with an object elicited larger N200-500 amplitudes than did repeating the novel word. In the After-training block, ERPs to the novel-paired words showed significantly larger negative mean area than did the novel words that were simply repeated (Fig. 3., ANOVA for the After-training block, Word experience, $F(1,20)$ $\left.=21.70, P=0.0002, \eta^{2}=0.52\right)$. These ERP effects tended to be larger over anterior than posterior regions (Word experience $\times$ Electrode site, $F(3,60)=3.39, P=0.038, \eta^{2}=0.15$ ).

\subsection{Group differences}

These analyses reflect main effects and interactions with language group from the ANOVAs described above. Group differences provide the primary test of our contrasting 


\section{NOVEL WORDS \\ BEFORE TRAINING}

\section{Left Hemisphere}
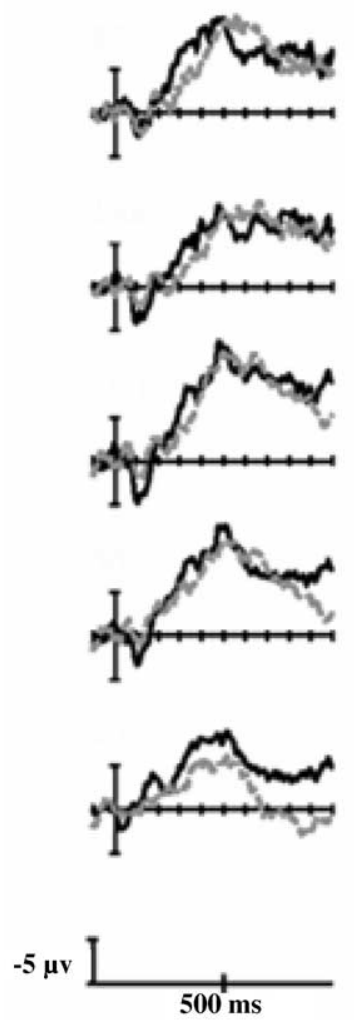

Right Hemisphere

Frontal

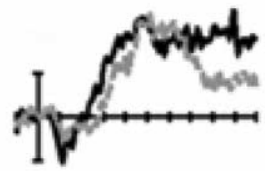

Ant Temporal

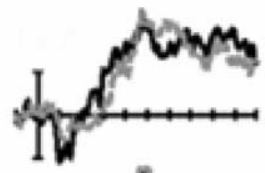

Temporal

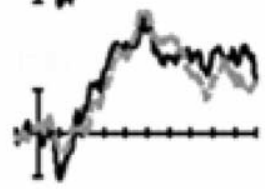

Parletal

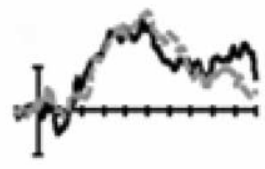

Occipital

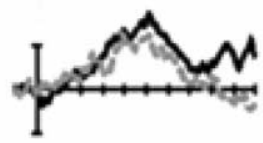

To be paired

Not to be paired

Fig. 2. ERPs to novel words before training.

hypotheses. If vocabulary size is the primary determinant of a left hemisphere specialization found in the 20-month-olds in the Mills et al. (1997) study, then the high producers should show ERP differences between the newly learned (novel-paired) and simply repeated (novel-not-paired) words that are limited to the left hemisphere. In contrast, if experience with the individual words is the determinant of increasing hemispheric specialization, then both groups should show bilateral differences between the newly learned and repeated words, as did the 13- to17-month-olds in the Mills et al. (1997) study.

\subsubsection{Before training}

Prior to training the high and low producers differed in the lateral distribution of the N200-500 to familiar words (familiar words: Language Group $\times$ Hemisphere $\times$ Electrode site, $F(3,60)=5.01, P=0.008, \eta^{2}=0.20$ ). That is, for the high producers, but not the low producers, the N200-500 was larger over the left than the right hemisphere over anterior 


\section{NOVEL WORDS \\ AFTER TRAINING}

Left Hemisphere
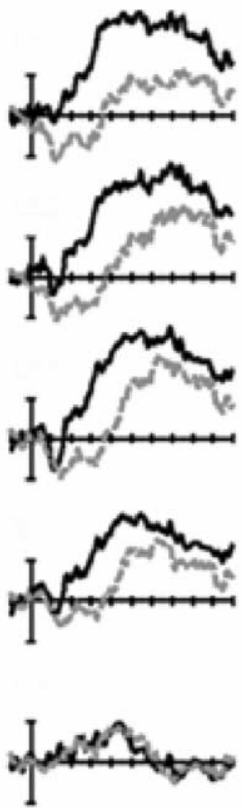

$-5 \mu \mathrm{v}$

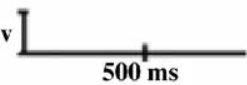

Right Hemisphere

Frontal

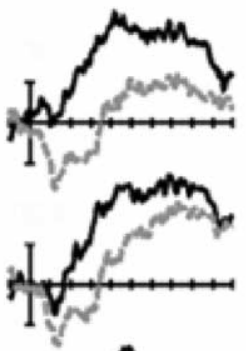

Temporal

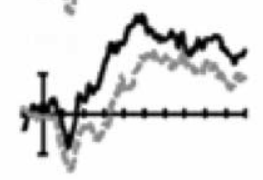

Parletal

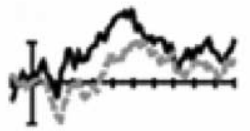

Occipital

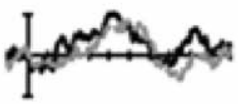

PAIRED

Not-paired

Fig. 3. ERPs to paired and not paired novel words after training.

regions [Hemisphere $\times$ Electrode site: High Producers, $F(3,60)=4.88, P=0.014, \eta^{2}=$ 0.20 ; Low Producers, $F(3,60)=0.64, P=0.56]$.

\subsubsection{After training}

Planned comparisons showed that for both the High and Low Producer groups, the N200-500 mean area was larger to the novel-paired words than novel-not-paired words (Word experience: for the High Producers, $F(1,20)=113.28, P=0.004, \eta^{2}=0.40$; for the Low Producers, $\left.F(1,20)=8.52, P=0.009, \eta^{2}=0.30\right)$. Consistent with the word experience hypothesis, for both groups ERP differences between novel-paired and novel-not-paired words were significant over both the left and right hemispheres (Word experience: High Producers, left hemisphere, $F(1,20)=17.43, P<0.0005, \eta^{2}=0.47$, right hemisphere, $F(1$, $20)=6.88, P=0.016, \eta^{2}=0.26$; Low Producers, left hemisphere, $F(1,20)=6.80, P=0.017$, $\eta^{2}=0.25$, right hemisphere, $\left.F(1,20)=7.95, P=0.011, \eta^{2}=0.28\right)$. Of particular interest for testing the word experience versus vocabulary size hypotheses, was whether a larger 


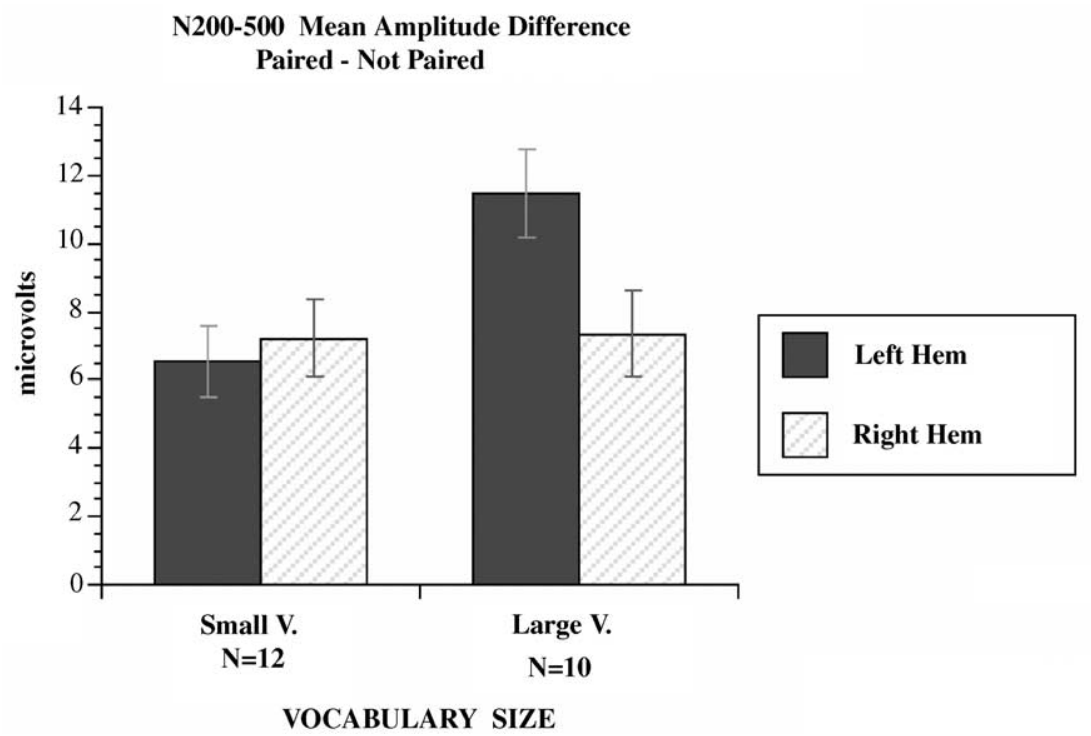

Fig. 4. Vocabulary size and distribution of training effect for High and Low Producers.

vocabulary size would also be linked with a left lateral organization of ERP differences to the novel-paired versus novel-not-paired words after training. There was a trend in that direction, but the effects failed to reach significant levels (Language Group $\times$ Word experience $\times$ Hemisphere, $\left.F(1,20)=2.99, P=0.10, \eta^{2}=0.13\right)$. Planned comparisons showed that for the High Producer group, these ERP amplitude differences were larger over the left than the right hemisphere (Fig. 4; High Producers, Word experience $\times$ Hemisphere, $F(1,20)=$ $\left.3.99, P=0.059, \eta^{2}=0.17\right)$.

\section{Discussion}

Our experiment set out to test the competing hypotheses that experience with individual word-object associations, or overall vocabulary size, is responsible for the dynamic shifts in ERP signatures observed in the original Mills et al. (1997) study. The results confirmed the former hypothesis: Both the low and high vocabulary groups showed ERP differences between novel-paired and novel-not-paired words which were bilateral and broadly distributed over anterior and posterior regions. The findings for the low vocabulary group parallel those reported for the 13-month-olds in Mills et al. study. However, the findings for the high vocabulary group contrast with Mills et al. 20-month-olds where the effects were limited to temporal and parietal regions of the left hemisphere. Mills et al. (1997) contrasted known with unknown words whereas we contrasted novel-paired with novelnot-paired words. We suggest that the relative familiarity of the stimuli in the two studies may account for the different pattern of results. In particular, the high vocabulary group's limited experience with novel-paired words resulted in bilateral as opposed to unilateral 
ERP difference effects. Therefore, our experiment indicates that individual experience with words is an important factor in determining the patterns of brain activity invoked during word processing: As the infant ages, her experience with individual words accrues, and this leads to increased cerebral specialization in the brain activity associated with these words.

Also of importance was the finding that object-novel word pairings led to an increase in N200-500 amplitudes, in contrast to simply repeating the novel words, which led to decreased N200-500 amplitudes. These findings provide strong support for the hypothesis that the N200-500 is linked to word meaning. The increased N200-500 cannot be due to differences in phonological familiarity because the novel-paired and novel-not-paired words were each heard the same number of times. Behavioral studies have shown that infants in this age range engage in fast mapping of word object pairings in as few as nine pairings (Woodward, Markman, \& Fitzsimmons, 1994; Schafer \& Plunkett, 1998). We cannot say definitively that the infants learned both new words. However, the repeated association of the object in the presence of the novel word was sufficient to elicit an increase in brain activity relative to the presence of the puppet and word without the presence of an object. Based on previous work using a similar training procedure and stimuli (Schafer \& Plunkett, 1998), we believe that the infants formed new word-object associations during the experiment.

Several aspects of our results warrant further consideration. First, the comparison of ERPs for familiar and novel words prior to training showed no systematic differences. This appears to be an anomalous result since familiar meaningful words are reported to yield negative ERPs for children in this age range (Mills et al., 1993, 1997; Mills, Conboy, et al., in press; Mills, Prat, et al., in press). It will be recalled, however, that the auditory stimuli in the current experiment were presented using infant-directed speech (IDS). Recent work (Zangl \& Mills, 2001; Zangl \& Mills, submitted for publication) found that IDS serves to boost the neural activity to unknown but not familiar words at 20 months of age. In contrast, thirteen-month-olds exhibited greater negativity for IDS compared to adult-directed speech (ADS) when presented with both familiar and unfamiliar words. Their interpretation of the results was that IDS serves to increase attention to the most relevant linguistic patterns given the infants' age and vocabulary size. At 20-months, hearing an unknown word in IDS boosts neural activity to that word. However, for well-known words, IDS has no advantage, and no increased neural activity over ADS. Direct comparisons between known and unknown words showed ERP amplitude differences for ADS, but not IDS (Zangl \& Mills, 2001). These results demonstrate that ERPs are sensitive to the manner in which linguistic stimuli are presented, even when those stimuli are identical words. Zangl and Mills' finding may go some way to explaining the absence of systematic differences between familiar and novel words in the pre-training phase of the current study. Since our stimuli were presented in IDS, novel words but not familiar words would attract preferential processing, thereby masking effects of semantic processing of familiar words.

A strict interpretation of the familiarity account predicts that the low and high vocabulary groups in our study should exhibit identical patterns of bilateral ERP differences for novel-paired versus novel-not-paired words. Indeed, a strict interpretation of our statistical analysis yielded exactly this finding. However, examination of Fig. 4 suggests some lateral asymmetry in the ERP signatures for the high vocabulary group. Differences between novel-paired and novel-not-paired words are greater in the left-hemisphere than the righthemisphere. The statistical analysis of this asymmetry revealed a non-significant trend. The 
small sample size may have contributed to the lack of statistical significance. A positive interpretation of this trend suggests an increasing cerebral specialization even for recently acquired words in the high vocabulary 20 -month-olds. In this regard, our results are not inconsistent with the proposal for a qualitative shift in strategy for processing known words with increasing vocabulary size as suggested by Mills et al. (1997). However, given the dominant role attributed to individual word experience in this study, we suggest a more parsimonious interpretation. By implication, the high vocabulary group has a greater rate of vocabulary growth than the low vocabulary 20 -month-olds. This group difference may reflect an increased efficiency in the manner in which some infants process familiar versus novel words, or perhaps a more general difference in learning rate between infants. Likewise, rate of acquisition may be important in shaping specializations such that cerebral asymmetries emerge more rapidly when learning itself is more rapid. In this sense, the emergence of lateral asymmetries, as observed here in the high vocabulary group, provides a snapshot of the brain in the process of becoming more specialized for language. According to this hypothesis, the lower vocabulary group would also show lateral differences to comprehended and unknown words after more exposure. On this interpretation, the dynamics of emerging cerebral specialization for processing words will depend not only on the amount of exposure to individual lexical items but also on other factors that influence the rate at which individual infants acquire a lexicon. All in all, our data suggest that quantitative factors, such as rate of acquisition and amount of exposure to individual words, may be adequate to account for dynamic shifts in ERP activity observed in children as they acquire their mental lexicon.

\section{Acknowledgement}

This research was supported by NIH grant DC0048111 from NIDCD to Helen Neville.

\section{References}

Conboy, B. (2002). Patterns of language processing and growth in early English-Spanish bilingualism. Doctoral dissertation, University of California San Diego and San Diego State University, 2000. Dissertation Abstracts International, B: The Sciences \& Engineering 63(11-B) (UMI No. 5193).

Conboy, B., \& Mills, D. L. (2000). Cerebral specialization for language comprehension in bilingual toddlers. Cognitive Neuroscience Society 200 Annual Meeting Abstracts. Journal of Cognitive Neuroscience, 17(Supplement), Abstract.

Corrigan, R. (1978). Language development as related to stage 6 object permanence development. Journal of Child Language, 5, 173-189.

Fenson, L., Dale, P. S., Reznick, J. S., Thal, D., Bates, E., Hartung, J., Pethick, S., \& Reilly, J. (1993). The MacArthur Communicative Development Inventories: User's guide and technical manual. San Diego, CA: Singular Publishing Group.

Gopnik, A., \& Meltzoff, A. (1987). The development of categorization in the second year and its relation to the other cognitive and linguistic developments. Child Development, 58, 1523-1531.

McShane, J. (1979). The development of naming. Linguistics, 17, 879-905.

Mills, D. L., Coffey-Corina, S. A., \& Neville, H. J. (1993). Language acquisition and cerebral specialization in 20-month-old infants. Journal of Cognitive Neuroscience, 5, 317-334. 
Mills, D. L., Coffey-Corina, S., \& Neville, H. J. (1997). Language comprehension and cerebral specialization from 13 to 20 months. Developmental Neuropsychology, 13, 397-445.

Mills, D. L., Conboy, B., \& Paton, C. (in press). Do changes in brain organization reflect shifts in symbolic functioning? In L. Namy (Ed.) Symbol Use and Symbolic Representation. Mahwah, NJ: Lawrence Erlbaum Associates.

Mills, D., Prat, C., Stager, C., Zangl, R., Neville, H., Werker, J. (in press). Language experience and the organization of brain activity to phonetically similar words: ERP evidence from 14- to 20-month olds. Journal of Cognitive Neuroscience.

Plunkett, K. (1993). Lexical segmentation and vocabulary growth in early language acquisition. Journal of Child Language, 20, 43-60.

Plunkett, K., Sinha, C., Møller, M. F., \& Strandsby, O. (1992). Symbol grounding or the emergence of symbols? Vocabulary growth in children and a connectionist net. Connection Science, 4, 293-312.

Rugg, M. D., \& Coles, M. G. H. (1995). Electrophyisology of Mind. Oxford: Oxford University Press.

Schafer, G., \& Plunkett, K. (1998). Rapid word learning by fifteen-month-olds under tightly controlled conditions. Child Development, 6, 309-320.

Woodward, A. L., Markman, E. M., \& Fitzsimmons, C. M. (1994). Rapid word learning in 13- and 18-month-olds. Developmental Psychology, 30, 553-566.

Zangl, R., \& Mills, D. L. (2001). The role of infant-directed speech in early language processing: An ERP-study. In Poster Society for Research in Child Development Conference.

Zangl, R., Mills, D. L. (submitted for publication). Increased brain activity to infant-directed speech in 6- and 13-month old infants. 